\title{
Emerin knockdown induces the migration and invasion of hepatocellular carcinoma cells by upregulating the cytoplasmic p21
}

\author{
Ke-Yan WU ${ }^{1,2, *}$, Hua XIE ${ }^{1, *}$, Zhi-Lin ZHANG ${ }^{1, *}$, Zi-Xian LI $^{1}$, Lin SHI ${ }^{1}$, Wei ZHOU ${ }^{1,2}$, Jing ZENG ${ }^{1}$, Zhen TIAN ${ }^{1}$, Yu ZHANG ${ }^{1}$, Yan-Bing DING ${ }^{2, *}$, \\ Wei-Gan SHEN ${ }^{1,3,4, *}$ \\ ${ }^{1}$ Department of Cell Biology, School of Medicine of Yangzhou University, Yangzhou, Jiangsu, China; ${ }^{2}$ Department of Gastroenterology, Affiliated \\ Hospital of Yangzhou University, Yangzhou, Jiangsu, China; ${ }^{3}$ Jiangsu Co-Innovation Center for Prevention and Control of Important Animal \\ Infectious Diseases and Zoonoses, Yangzhou University, Yangzhou, Jiangsu, China; ${ }^{4}$ Jiangsu Key Laboratory of Integrated Traditional Chinese \\ and Western Medicine for Prevention and Treatment of Senile Diseases, Yangzhou University, Yangzhou, Jiangsu, China
}

*Correspondence: ybding@yzu.edu.cn; shenweigan@hotmail.com

"Contributed equally to this work.

Received July 28, 2021 / Accepted October 1, 2021

\begin{abstract}
Emerin (EMD) plays diverse roles in cellular polarity organization, nuclear stability, and cell motility, however, the biological role of EMD relevant to the migration and invasion of hepatocellular carcinoma (HCC) cells has not yet been illustrated. In the present study, we initially found that the upregulation of EMD in HCC tissues, and EMD expression was negatively correlated with the spontaneous metastatic potential of HCC cell lines. Loss of EMD in HCC cells facilitated cell migration and invasion in vitro and metastasis in vivo. Meanwhile, we demonstrated that EMD knockdown induced EMT but enhanced p21 expression in HCC cells. Notably, silencing of EMD in HCC cells increased the cytoplasmic localization of $\mathrm{p} 21$ protein, whereas $\mathrm{p} 21$ knockdown partially abrogated the migratory and invasive ability, EMT, and the actin cytoskeleton rearrangement induced by EMD knockdown in HCC cells. Our results indicated a significant role of EMD knockdown in the HCC cell motility and metastasis through upregulating the cytoplasmic p21, unveiling a novel mechanism of cell motility regulation induced by EMD.
\end{abstract}

Key words: Emerin, hepatocellular carcinoma cell, p21, migration, invasion

Hepatocellular carcinoma (HCC) is the sixth most prevalent malignancy with more than 800,000 patients' mortalities annually worldwide $[1,2]$. Although recent advances in surgical resection, liver transplantation, radiofrequency ablation, and novel drug therapy, the five-year survival rates for the HCC patients remained less than $20 \%$ due to the high frequency of tumor recurrence and distance metastasis $[3,4]$. Therefore, it is imperative to further explicate the molecular mechanisms underlying HCC metastasis to develop novel therapeutic strategies and to identify the prognostic biomarkers.

Emerin (EMD) belongs to a family of the nuclear laminaassociated proteins, and it is widely expressed in the inner nuclear envelope and plays diverse roles in cellular polarity organization, nuclear stability, chromatin tethering, gene expression, cell signaling, and mechanotransduction [5-8]. In EMD-deficient cells, nuclear elasticity decreases, nuclear fragility increases, and nuclear membrane is more plastic [9]. Additionally, EMD can promote actin polymerization by binding and stabilizing the pointed end of growing actin filaments [10], and EMD deletion promotes nuclear instability in prostate cancer, lung cancer, and thyroid cancer cells $[5,11$, 12], thereby promoting tumor invasion and metastasis. Nevertheless, the biological role of EMD relevant to the migration and invasion of HCC cells has not yet been illustrated.

In the current study, the upregulation of EMD in HCC tissues was first identified. We further demonstrated that EMD was differentially expressed in HCC cell lines with different spontaneous metastatic potential, and EMD knockdown promoted the migratory ability and invasiveness of HCC cells in vitro and in vivo. Strikingly, we verified that EMD knockdown induced epithelial-mesenchymal transition (EMT) by upregulating the cytoplasmic p21 in HCC cells. This finding might provide interesting mechanism for the HCC metastasis and identify EMD as a potential clinically relevant biomarker of tumor aggressiveness.

\section{Materials and methods}

Cell culture, transfection, and reagents. The human HCC cell lines (HCC-LM3, MHCC-97H, SMMC-7721, 
SK-HEP1, Hep3B, and HepG2) and human embryonic kidney cell line 293T (HEK293T) were obtained from the Type Culture Collection of the Chinese Academy of Sciences (Shanghai, China). All the cell lines in the present study were regularly verified for the absence of mycoplasma contamination, and cultured in DMEM (Hyclone, Beijing, China) with $10 \%$ fetal bovine serum (FBS, Gibco, USA) and $1 \%$ penicillin-streptomycin at $37^{\circ} \mathrm{C}$ in a humidified incubator with $5 \% \mathrm{CO}_{2}$. Control and p21-specific siRNAs were obtained from RIBOBIO Company (Guangzhou, China). Transfection of siRNA into HCC cells was performed using jetPRIME Polyplus kit (France) according to the manufacturer's instructions.

Tissue microarray (TMA) and immunohistochemistry. HCC TMA with 103 cases of the HCC tissues and matched adjacent non-tumor liver tissues were acquired as previously described [13]. Immunohistochemistry (IHC) staining of EMD on the TMAs was performed using an IHC detection kit obtained from Servicebio Technology (Wuhan, China) according to the manufacturer's instructions. Briefly, after rehydration and antigen retrieval, the TMAs were incubated with the monoclonal antibody against EMD (\#sc-81552, Santa Cruz Biotechnology, USA) at $4{ }^{\circ} \mathrm{C}$ overnight, followed by the incubation with the secondary antibody (\#sc-2089, Santa Cruz Biotechnology, USA) at room temperature for $1 \mathrm{~h}$. The slides were stained with prepared diaminoaniline (DAB) and counterstained with hematoxylin. The areal density of each HCC case in TMAs was analyzed by Aperio Digital Pathology Slide Scanners (Servicebio, Wuhan, China).

Plasmid construction, lentivirus production, and infection. The short hairpin RNA (shRNA) targeting EMD (shEMD1, sense, 5'-GATCCGAGGTGCATGATGACGATCT T TCAAGAGAAGATCGTCATCATG CACCTCTTTTTTA-3 and antisense, 5'-CGCGTAAAAAAGAGGTGCATGATGACGATCTTCTCTTGAAAGATCGTCATCATGCACCTCG-3'; shEMD2, sense, 5'-GATCCGGCTTCAGAGCTAGGTCTTTGTTCAAGAGACAAAGACCTAGCTCTGAAGCCTTTTTTA-3' and antisense, 5'-CGCGTAAAAAAGGCTTCAGAGCTAGGTCTTTGTCTCTTGAACAAAGACCTAGCTCTGAAGCCG-3') and the negative control shRNA (shNC, sense, 5'-GATCCTGGTTTACATGTCGACTAATTCAAGAGATTAGTCGACATGTAAACCATTTTTTA-3' and antisense, 5' - CGCGTAAAAAATGGTTTACATGTCGACTAATCTCTTGAATTAGTCGACATGTAAACCACG-3') were cloned into the BamH I and Mlu I sites of the pLentU6-Puro vector (ViGene Biosciences Inc., Rockville, MD, USA). Lentiviral particles were packaged using HEK293T cells by transfection with shNC or shRNA targeting EMD constructs together with the lentiviral packaging vector pSPAX2 and pMD2G (Addgene) using EndoFectin Lenti reagent (GeneCopoeia, USA). Virus-containing supernatants were harvested and filtered. SMMC-7721 and HCC-LM3 cells were transduced with the lentiviruses in the presence of polybrene $(8 \mathrm{mg} / \mathrm{ml})$ for $48 \mathrm{~h}$ before selection with puromycin $(0.5 \mu \mathrm{g} / \mathrm{ml}$ for SMMC-7721 and $0.4 \mu \mathrm{g} / \mathrm{ml}$ for HCC-LM3 cells). Stable EMD knockdown cell lines were screened by reverse transcription-quantitative polymerase chain reaction (RT-qPCR) and western blotting.

Cell proliferation assay. HCC cells (5,000/well) were seeded in 96-well plates with eight duplications. Cell viability was determined using the Cell Counting Kit-8 (CCK-8) kit (SunBio Biomedical Technology, Shanghai, China). Briefly, $10 \mu \mathrm{l}$ of the CCK- 8 solution was added to each well and incubated at $37^{\circ} \mathrm{C}$ for $45 \mathrm{~min}$. Optical density was detected at a wavelength of $450 \mathrm{~nm}$ using a microplate reader (Thermo, USA). A 5-ethynyl-20-deoxyuridine (EdU) assay kit (Ribobio, Guangzhou, China) was performed to evaluate the cell proliferation ability according to the manufacturer's instructions. For cell cycle analysis, HCC cells were harvested and fixed with chilled 75\% ethanol, and cell cycle analysis was determined using PI kits (Solarbio Science \& Technology, Beijing, China) on the flow cytometry according to the manufacturer's instructions. All experiments were performed at least in triplicate.

RNA-sequencing (RNA-seq) and RT-qPCR. Total RNA was extracted using the TRIzol $^{\circ}$ reagent (Invitrogen, USA). RNA-seq analysis was performed by Wuhan Igenebook Co., Ltd. (Wuhan, China). For RT-qPCR, reversetranscription was performed using the PrimeScriptTM RT Master Mix (Takara Biotechnology, Co., Ltd., Dalian, China), and qPCR was carried out using the SYBR Green qPCR system (Takara Biotechnology, Co., Ltd., Dalian, China) on a LightCycler system (Mannheim, Germany). Primers used in the present study were as follows: $\mathrm{N}$-cadherin (N-CAD) forward, 5'-CAGTGTACAGAATCAGTG-3' and reverse, 5'-CAACAGTAAGGACAA ACA-3'; E-cadherin (E-CAD) forward, 5'-CAAGTGACCACCTTAGAG-3' and reverse, 5'-GAATTTGCAATCCTGCTT-3'; Vimentin (VIM) forward, 5'-GACGCCATCAACACCGAGTT-3' and reverse, 5'-CTTTGTCGTTGGTTAGCTGGT-3'; p21 forward, 5'-GGCCCAGTGGACAGCGAGCA-3' and reverse, 5'-CCCAGGCGAAGT CACCCTCC-3'; GAPDH forward, 5'-GCACCGTCAAGGCTGAGAAC-3' and reverse, 5'-TGGTGAAGACGCCAGTGGA-3'. The relative expression of the mRNAs was calculated using the $2^{-\Delta \Delta C t}$ method with GAPDH as an endogenous control.

Cell migration and invasion assays. Transwell inserts (diameter, $6.5 \mathrm{~mm}$; pore size, $8.0 \mu \mathrm{m}$; Corning, NY, USA) were used to perform cell migration and invasion assays as described previously [13]. Briefly, HCC cells were serumstarved overnight, and then SMMC-7721 $\left(3 \times 10^{4}\right)$ or HCC-LM3 $\left(2 \times 10^{4}\right)$ cells in $200 \mu$ l of DMEM without FBS were seeded into the uncoated or Matrigel-coated upper chambers. The inserts were then placed into the lower chambers with $600 \mu \mathrm{l}$ of DMEM containing $10 \% \mathrm{FBS}$ and incubated at $37^{\circ} \mathrm{C}$ for $24 \mathrm{~h}$. The cells penetrating the membrane were fixed and stained with crystal violet, and the phase-contrast images were captured and five random fields at 200× magnification were analyzed. 
Nuclear/cytoplasmic fractionation. Nuclear and cytoplasmic protein extraction from HCC cells was performed using the Nuclear and Cytoplasmic Extraction kit (Solarbio Science \& Technology, Beijing, China) according to the laboratory manual with GAPDH and Histone H3 expression as loading controls for cytosolic and nuclear fraction, respectively.

Western blotting. Western blotting was performed following standard methods. Briefly, equal amounts of proteins were separated by $10 \%$ SDS-PAGE and transferred onto PVDF membranes. After blocking with 5\% non-fat milk powder in TBST for $2 \mathrm{~h}$, the membranes were incubated with primary antibodies overnight at $4{ }^{\circ} \mathrm{C}$ and then incubated with HRP-linked anti-rabbit or antimouse IgG for $2 \mathrm{~h}$. Signals were detected by ECL (Thermo Fisher Scientific, Inc.). The following antibodies were used in the present study: rabbit anti-Snail (\#3879), rabbit antiVimentin (\#5741), rabbit anti-p21 (\#2947), and HRP linked anti-rabbit IgG (\#7074) and anti-mouse IgG (\#7076) were purchased from Cell Signaling Technology (Danvers, MA, USA). The mouse anti-E-cadherin (\#562869) and mouse anti-N-cadherin (\#561553) were purchased from BD Biosciences (San Jose, CA, USA). The mouse anti-Emerin (\#sc-81552) was purchased from Santa Cruz Biotechnology (Texas, USA). The monoclonal antibody against GAPDH (\#abs137959) was purchased from Absin Bioscience (Absin, China).

Immunofluorescence analysis. Cells grown on glass coverslips in a 24 -well culture plate were fixed with $4 \%$ paraformaldehyde for $30 \mathrm{~min}$ and permeabilized with $0.1 \%$ Triton X-100 for $10 \mathrm{~min}$. After blocking with 3\% BSA in PBS, the cells were incubated with primary antibody against p21 (\#2947, Cell Signaling Technology, diluted 1:100 in PBS) at $4{ }^{\circ} \mathrm{C}$ overnight, followed by incubation with rhodamine-conjugated secondary antibody (\#sc-2492, Santa Cruz Biotechnology, diluted 1:500 in PBS) for $2 \mathrm{~h}$ at room temperature. For F-actin staining, the cells were incubated with the rhodamine-conjugated phalloidin (Sigma, USA) for $1 \mathrm{~h}$ at room temperature. All samples were washed with PBS, and the nuclei were labeled with DAPI (Beyotime, China). Images were obtained with an Eclipse E600 fluorescent microscope (Nikon, Japan).

Tail vein metastasis models. Athymic nude mice (BALB/C-nu/nu, 6-8 weeks old, male) were obtained from the Animal Centre of Yangzhou University and maintained in a pathogen-free environment. All animal procedures were performed in compliance with the institutional ethical requirements and were approved by the Yangzhou University School of Medicine Committee for the Use and Care of Animals. $1 \times 10^{6}$ cells resuspended in $100 \mu \mathrm{l}$ PBS were injected into the tail veins of the mice. All of the mice were sacrificed under anesthesia 24 days after the inoculation. Liver and lung tissues were removed, fixed in formalin, and embedded in paraffin. Consecutive sections were analyzed by hematoxylin and eosin (H\&E) staining.
Statistical analysis. Statistical analysis was performed with GraphPad Prism 7 software (San Diego, CA, USA). Data were presented as mean \pm standard deviation (SD). Two group comparisons were determined by using the Student's t-test, while multiple group comparisons were performed using the one-way ANOVA. A p-value less than 0.05 was considered statistically significant.

\section{Results}

The expression of EMD in HCC tissues and HCC cell lines. To clarify the role of EMD in HCC, we first performed IHC staining to detect the expression of EMD in 103 pairs of clinical HCC samples. TMA analysis showed that the positive EMD staining was mainly observed in the nuclear region in the HCC tissues and in the cytoplasm in the paracancerous tissues (Figure 1A). Density analysis of TMAs revealed that EMD expression in HCC tissues (T) was higher than that in corresponding paracancerous tissues (P) (Figure 1B). Meanwhile, data from The Cancer Genome Atlas (TCGA) database (https://genome-cancer. ucsc.edu) demonstrated that the mRNA expression of EMD was higher in HCC tissues (T) than those in normal tissues (P) (Figure 1C). In addition, we also examined the expression of EMD in HCC cell lines. The expression of EMD in HepG2, SMMC-7721, Hep3B, MHCC-97H, HCC-LM3, and SK-HEP1 cells, six HCC cell lines with different spontaneous metastatic potential, was measured by western blotting. As shown in Figure 1D, relative low expression of EMD was shown in MHCC97H, SK-HEP1, and HCC-LM3 cells with high metastatic potential, and abundant expression of EMD was shown in the HepG2, SMMC-7721, and Hep3B cells with low metastatic potential, which points to the connection between EMD and metastasis. Taken together, these findings suggest that EMD is upregulated in HCC and might be related to metastasis.

EMD knockdown promoted the migration and invasion of HCC cells in vitro and tumor metastasis in vivo. To explore whether EMD regulated HCC cell motility, we used the lentiviral-mediated gene knockdown approaches to silence the endogenous EMD in both SMMC-7721 and HCC-LM3 cells. The knockdown efficiency of EMD was confirmed in stable EMD knocking down SMMC-7721 and HCC-LM3 cells by RT-qPCR and western blotting (Figures 2A, 2B). Transwell assays demonstrated that, compared with the negative control (shNC), both SMMC-7721 and HCC-LM3 cells stably knocking down EMD showed a significant increase of the cell migratory and invasiveness capacity (Figures 3A, $3 \mathrm{~B})$. Additionally, the alteration in cell motility induced by EMD knockdown did not appear to be due to influencing the cell viability and proliferation. We demonstrated that EMD knockdown had no significant effects on cell viability, cell cycle progression, and proliferation of SMMC-7721 and HCC-LM3 cells in vitro (Figures $2 \mathrm{C}-2 \mathrm{G}$ ). Given that the dynam ic assembly of the actin cytoskeleton plays an impor- 


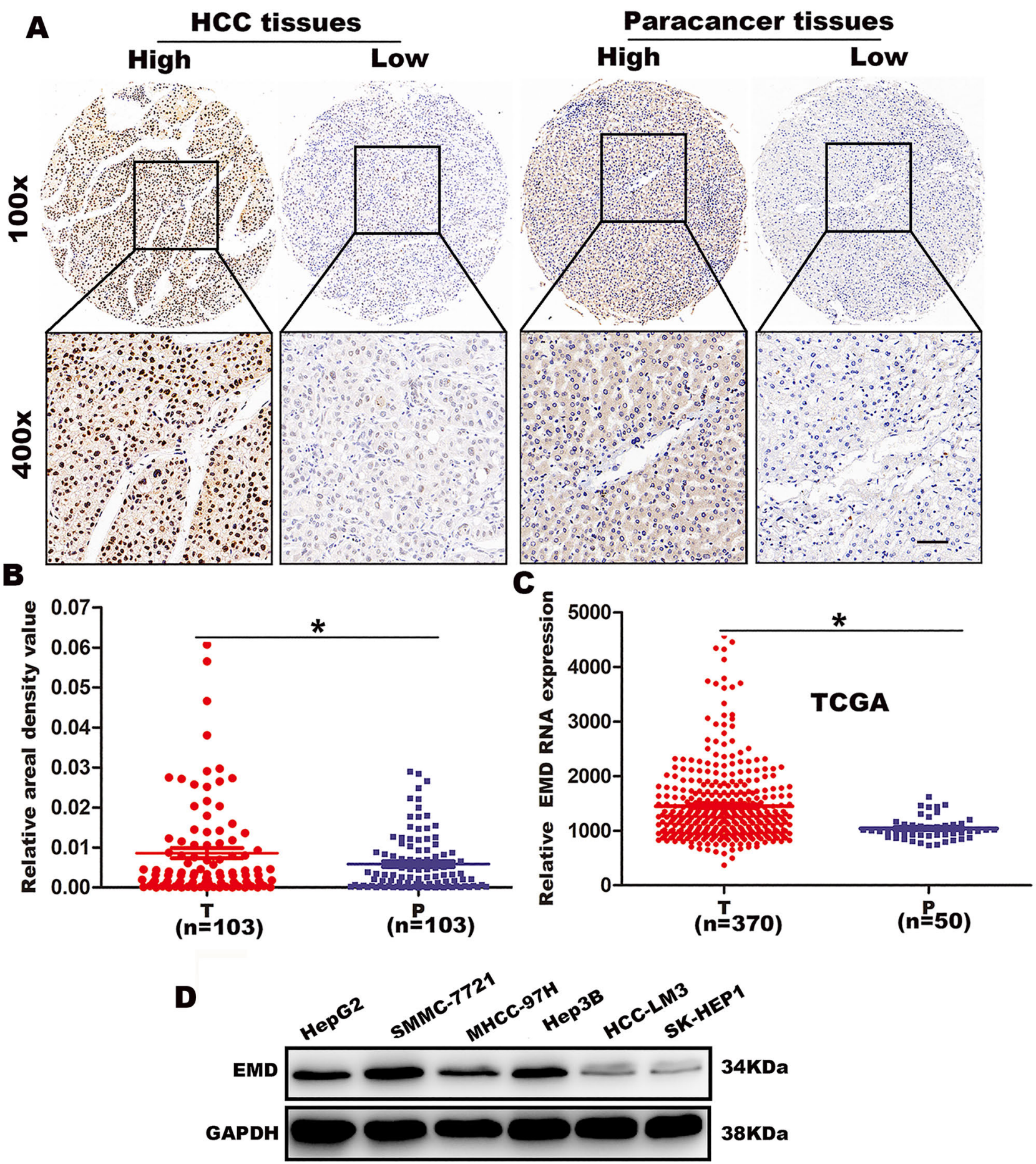

Figure 1. Expression of EMD in HCC tissues and HCC cell lines. A) Representative IHC images of EMD staining in TMAs of HCC tumor or adjacent tissues (magnification, 100 $\times$ and $400 \times$ ) are shown. Scale bar; $100 \mu \mathrm{m}$. B) IHC density analysis of 103 pairs of HCC tissues (T) and matched paracancerous tissues $(\mathrm{P})$ in the TMAs based on EMD staining is shown. Data presented as mean $\pm \mathrm{SD} .{ }^{*} \mathbf{p}<0.05$. C) Analysis of EMD mRNA levels in HCC tissues (T) and normal tissues (P) from the TCGA database (https://genome-cancer. ucsc.edu) is shown. ${ }^{\star} \mathbf{p}<0.05$. D) The expression of EMD in HCC cell lines HepG2, SMMC-7721, Hep3B, MHCC-97H, HCC-LM3, and SK-HEP1 with different spontaneous metastatic potential was detected by western blotting. GAPDH served as a loading control. Results represent three independent experiments. 
A

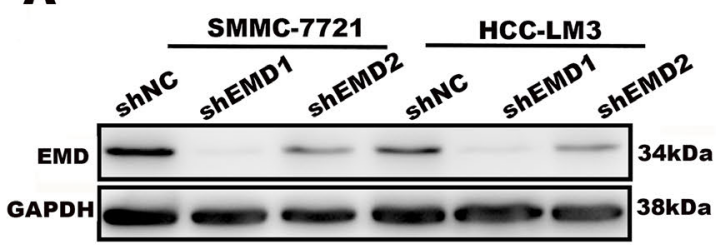

C

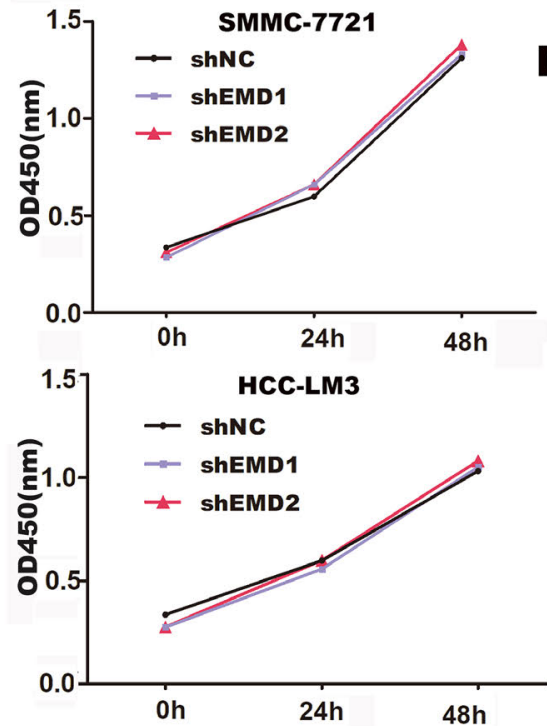

E

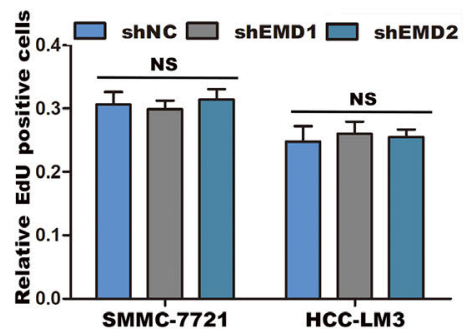

C

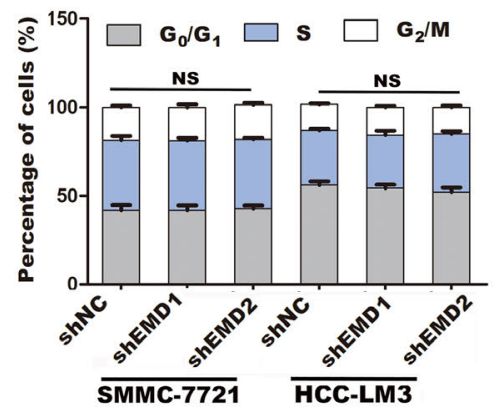

D

$\mathbf{F}$
B
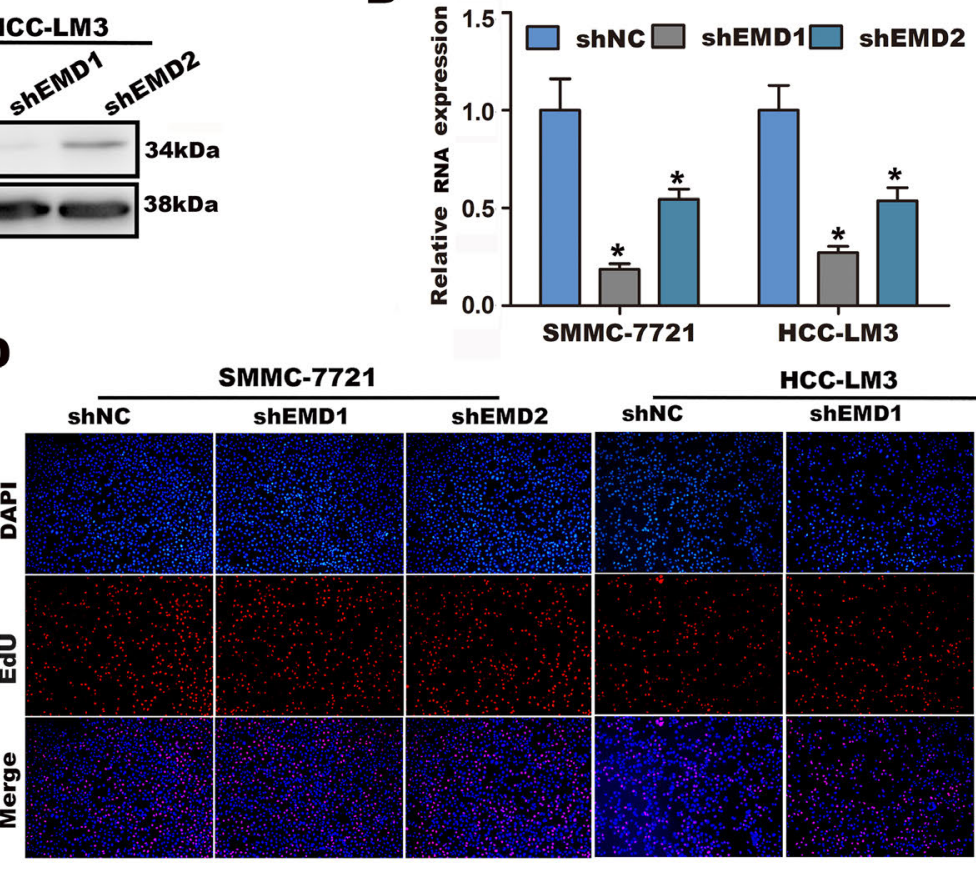

ShEMD1 ShEMD2

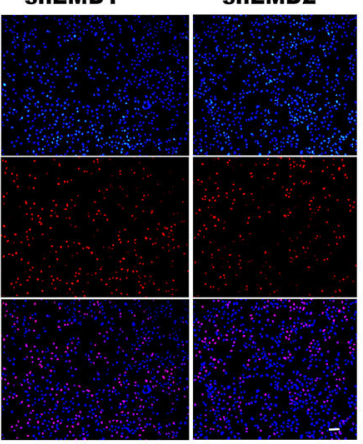

SMMC-7721
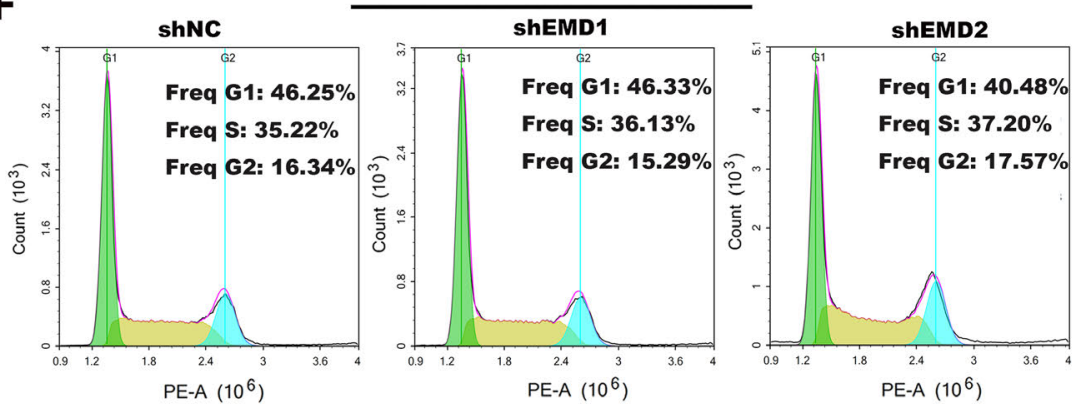

HCC-LM3
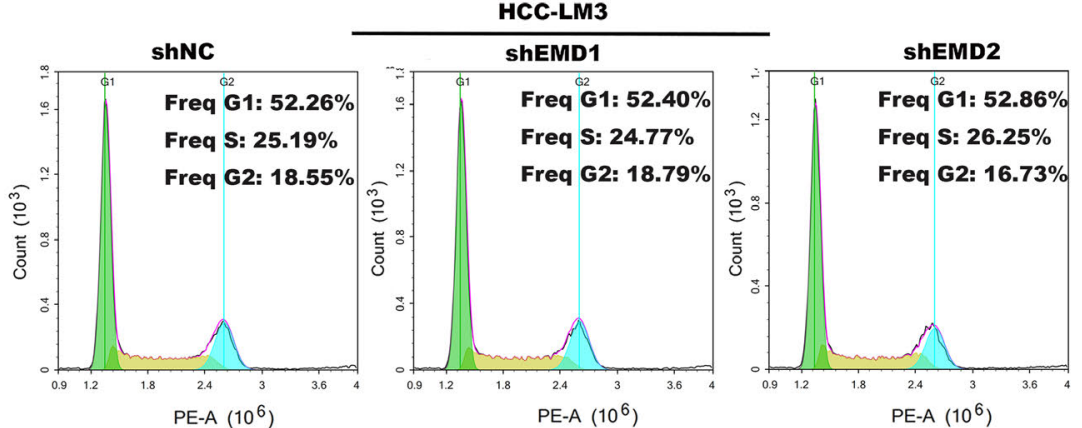

PE-A $\left(10^{6}\right)$

Figure 2. Effect of EMD knockdown on HCC cell proliferation. A, B) The lentiviral-mediated gene knockdown approaches to silence the endogenous EMD in both SMMC-7721 and HCC-LM3 cells, and the knockdown efficiency of EMD was tested by western blotting (A) and RT-qPCR (B) in stable EMD-knocked down SMMC-7721 and HCC-LM3 cells. GAPDH served as a loading control. Data are shown as the mean \pm SD of three independent experiments. ${ }^{*} \mathrm{p}<0.05$, vs. the indicated control (shNC). C) Cell viability of SMMC-7721 and HCC-LM3 cells was assessed by the CCK-8 assay. Data are shown as the mean \pm SD of three independent experiments. D, E) EdU assay was applied to compare the cell proliferation ability in stable EMDknocked down SMMC-7721 and HCC-LM3 cells and the indicated control cells (shNC) (D) (Scale bar; $100 \mu \mathrm{m}$ ). Quantification of relative EdU positive cells for SMMC-7721 and HCC-LM3 cells (E) is shown. Data presented as mean \pm SD of three independent experiments. F, G) Representative images of the cell cycle progression in stable EMD-knocked down SMMC-7721 and HCC-LM3 cells and the indicated control cells (shNC) (F) are shown, and the statistical analysis of the results to show the cell cycle progression $(G)$. Data presented as mean \pm SD of three independent experiments. 
tant role in cell motility [14], we further investigated whether silencing of EMD could modulate the actin cytoskeleton rearrangement in HCC cells. As shown in Figure 3C, fewer stress fibers and more lamellipodia at the leading edge, which are associated with an increase of cell motility, were shown in both EMD knocking down SMMC-7721 and HCC-LM3 cells. Collectively, these results indicated that EMD knockdown significantly accelerated the migration and invasion of SMMC-7721 and HCC-LM3 cells in vitro.

To further explore whether EMD plays a role in tumor metastasis, we assessed the effect of EMD knockdown on tumor metastasis in vivo. HCC-LM3 cells with stably knocked down EMD and the control cells were injected into the tail veins of nude mice to establish in vivo metastasis models. Twenty-four days later, nude mice were sacrificed and the tumor metastases were analyzed. The metastatic nodules in the livers and lungs were significantly increased in mice injected with HCC-LM3 cells stably knocking down EMD, compared to those injected with control cells (Figures 4A-4D). Hematoxylin-eosin staining showed that the metastatic foci derived from the HCC-LM3 cells stably knocking down EMD increased in the liver and lung sections (Figures 4E, 4F). Taken together, these findings suggest that the tumor metastatic potential of HCC cells was facilitated by the silencing of EMD.

EMD knockdown induced EMT but enhanced p21 expression in HCC cells. To gain insights into the regulatory molecular mechanism by which EMD knockdown enhanced
$\mathbf{A}$
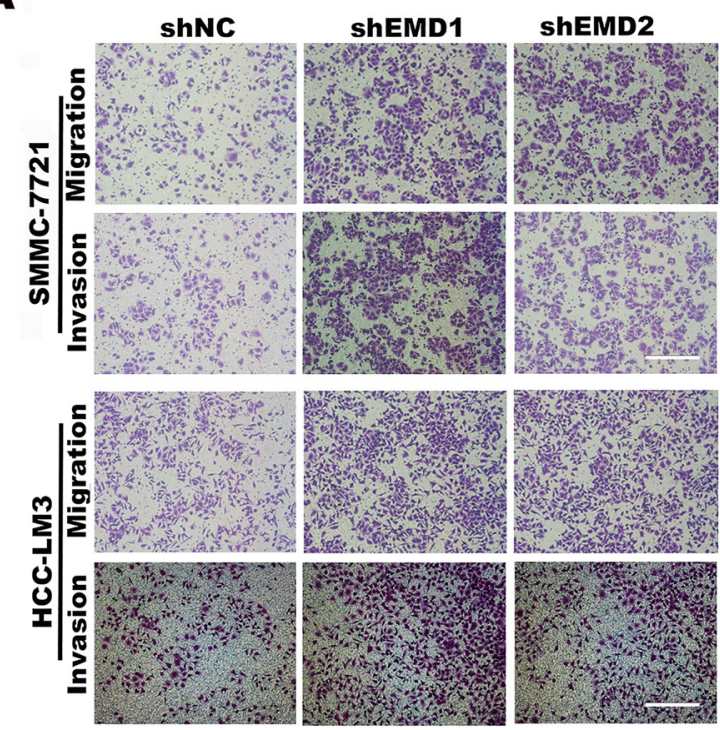

C

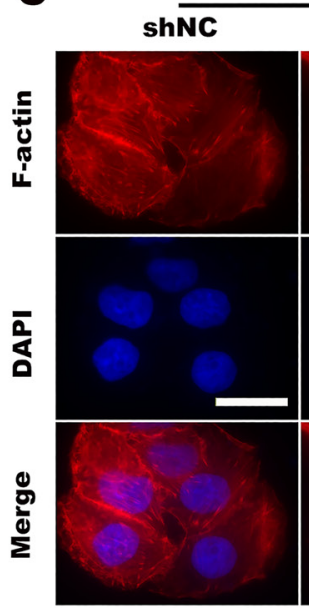

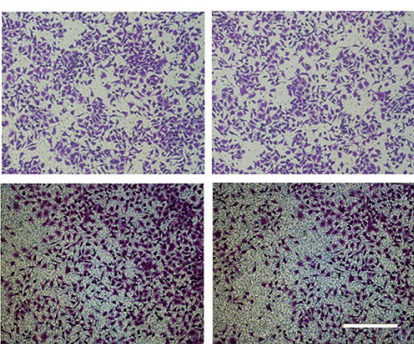

SMMC-7721 ShEMD2

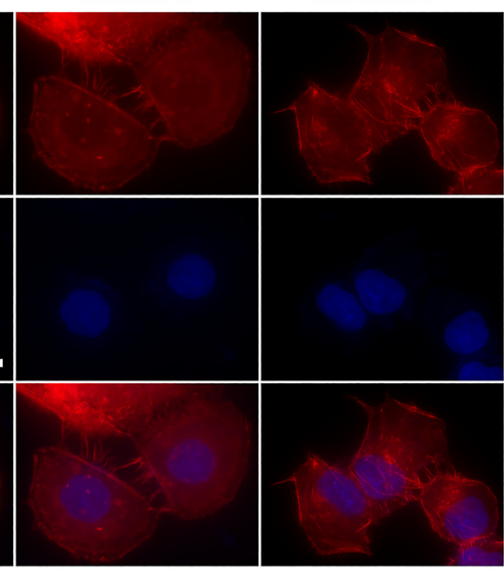

Figure 3. EMD knockdown promoted migration and invasion of HCC cells in vitro. A, B) Representative Transwell cell migration and invasion assays in EMD-knocked down SMMC-7721 and HCC-LM3 cells (A, scale bar; $100 \mu \mathrm{m}$ ), and quantification of relative numbers of migrated and invaded cells (B) is shown. Data presented as the mean \pm SD of three independent experiments. ${ }^{*} \mathbf{p}<0.05$, vs. the indicated control (shNC). C) Representative images of the actin cytoskeleton and DAPI staining in EMD-knocked down SMMC-7721 and HCC-LM3 cells are shown. Scale bar; $20 \mu \mathrm{m}$. 
A
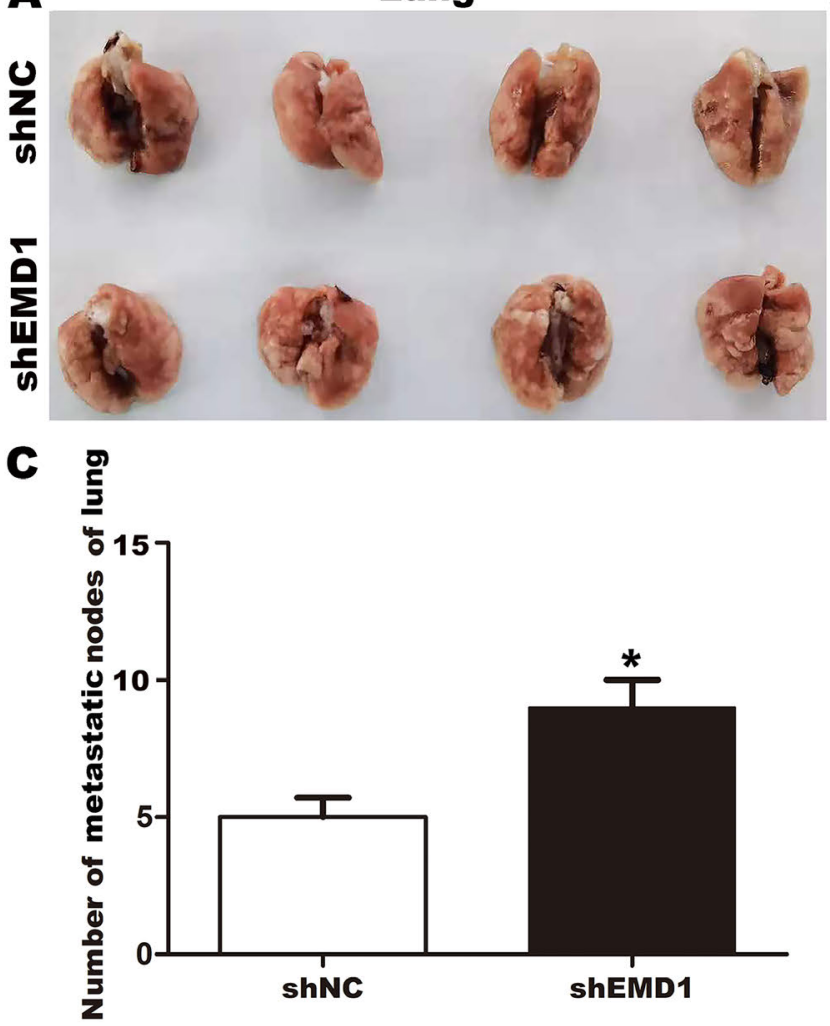

$\mathbf{E}$

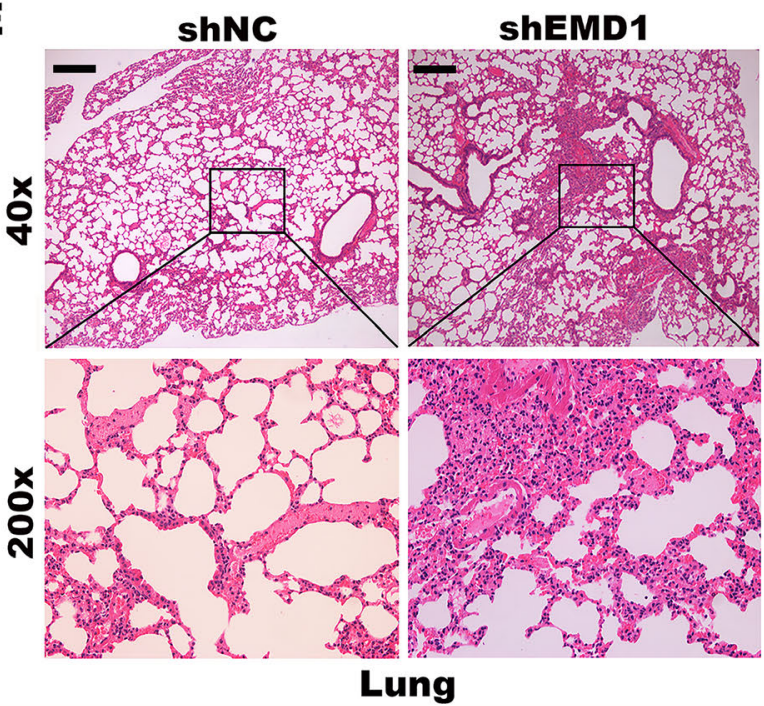

B

Liver

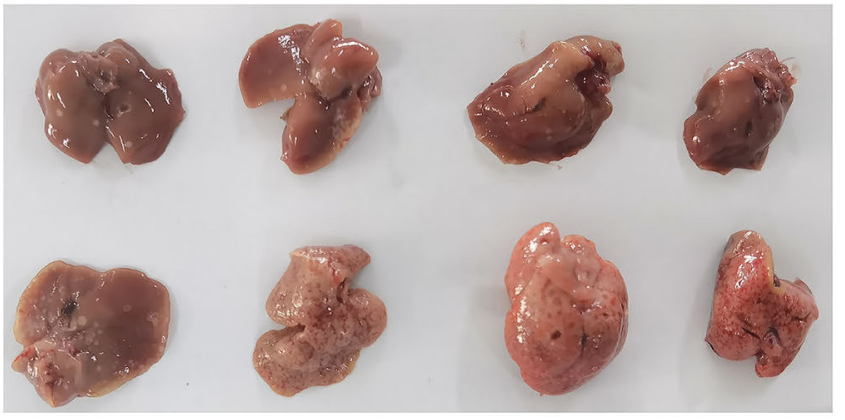

D

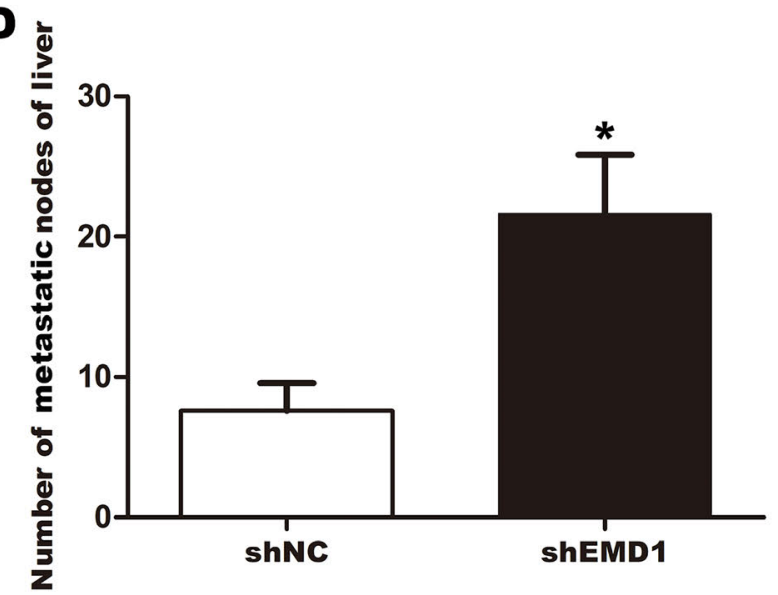

$\mathbf{F}$

shNC
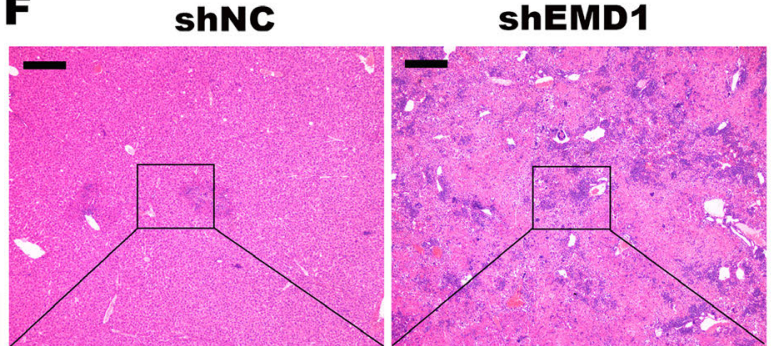

$\stackrel{x}{g}$

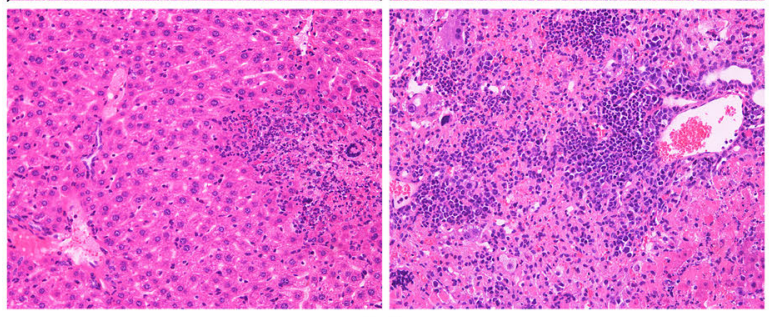

ڤั̀

Figure 4. EMD knockdown promoted tumor metastasis in vivo. A, B) Representative macroscopic appearance of the lungs (A) and livers (B) in nude mice after the tail veins injection with EMD-knocked down HCC-LM3 cells and shNC cells for 24 days (n=8). C, D) Quantification of the metastatic nodules in the lungs $(\mathrm{C})$ and livers $(\mathrm{D})$ is shown. Data presented as the mean $\pm \mathrm{SD} .{ }^{\star} \mathrm{p}<0.05$, vs. shNC. E, F) Representative H\&E staining images of the lung (E) and liver (F) tissue sections from indicated groups are shown. Scale bar; $100 \mu \mathrm{m}$.

the malignant phenotype of HCC cells in terms of comprehensive gene expression, RNA-seq was employed to explore the expression changes between EMD-knocked down SMMC-7721 cells and the control cells. As shown in Figure $5 \mathrm{~A}$, a total of 595 upregulated genes and 537 downregulated genes were differentially expressed by at least a 1.5-fold change in EMD-knocked down cells. Kyoto Encyclopedia of Genes and Genomes (KEGG) and Gene Ontology (GO) analysis showed that the regulation of cell motility and actin cytoskeleton was affected in EMD-knocked down 


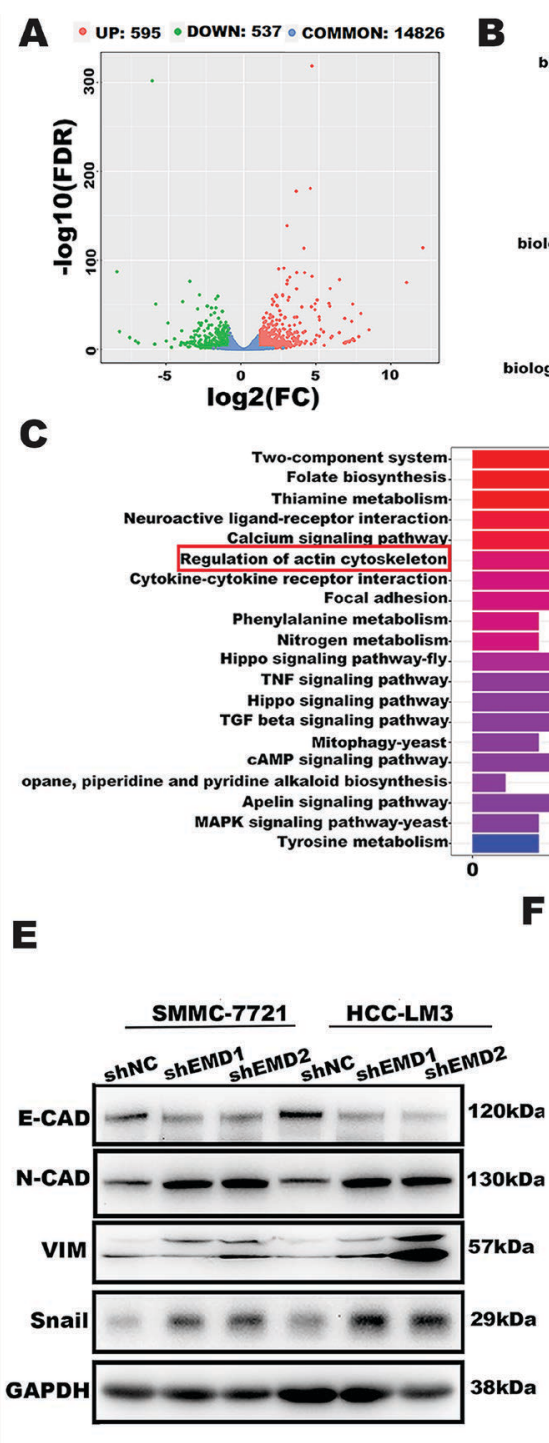

H

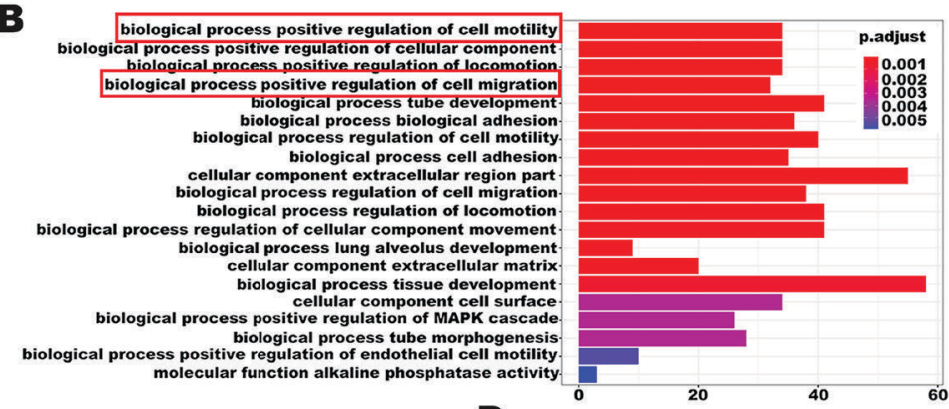

D
SMMC-7721

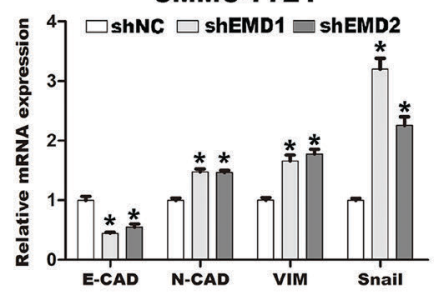

HCC-LM3

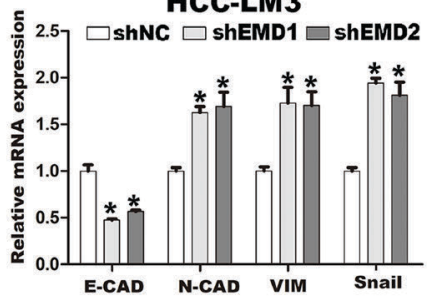

G

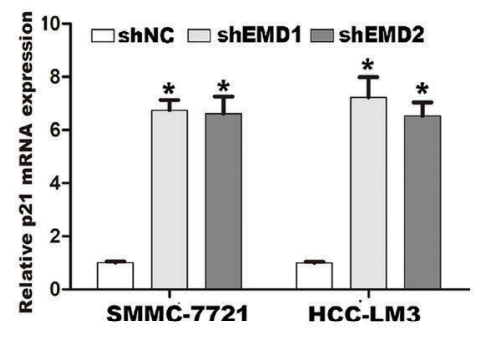

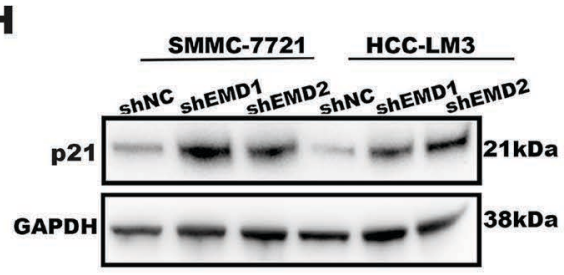
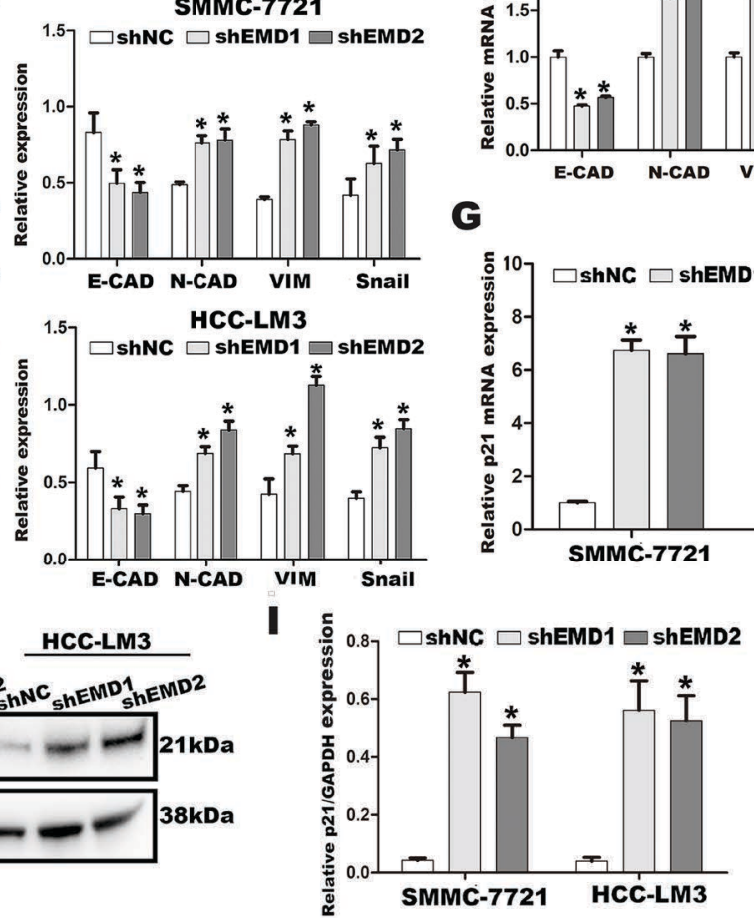

Figure 5. EMD knockdown induced EMT but enhanced p21 expression in HCC cells. A) Volcano plot of the differentially expressed genes identified in EMD-knocked down SMMC-7721 cells and control cells (both in triplicate) from RNA-seq analysis is shown. B, C) GO analysis (B) and KEGG analysis (C) revealed that the differentially expressed genes were enriched in the modulation of cell motility, actin cytoskeleton, and in various pathways. D) RT-qPCR was performed in EMD-knocked down SMMC-7721 and HCC-LM3 cells to validate the expression of E-cadherin (E-CAD), Vimentin (VIM), Snail, and $\mathrm{N}$-cadherin (N-CAD). GAPDH served as a loading control. Data are shown as the mean \pm SD of four independent experiments. ${ }^{\star} \mathrm{p}<0.05$, vs. the indicated shNC. E, F) Representative blots in EMD-knocked down SMMC-7721 and HCC-LM3 cells and control cells are shown, and quantification of the relative protein levels of EMT markers is shown (F). GAPDH served as a loading control. Data are shown as the mean \pm SD of three independent experiments. ${ }^{\star} \mathrm{p}<0.05$, vs. the indicated shNC. G) RT-qPCR analysis of p21 expression in EMD-knocked down SMMC-7721 and HCC-LM3 cells. GAPDH was used as an internal reference. Data are shown as the mean $\pm \mathrm{SD}$ of four independent experiments. ${ }^{*} \mathrm{p}<0.05$, vs. the indicated shNC. $\left.\mathrm{H}, \mathrm{I}\right)$ Protein levels of p21 were determined by western blotting in EMD-knocked down SMMC-7721 and HCC-LM3 cells, and quantification of the relative protein levels of $\mathbf{p} 21$ is shown (I). GAPDH served as a loading control. Data are shown as the mean \pm SD of three independent experiments. ${ }^{\star}$ p $<0.05$, vs. the indicated shNC. 
SMMC-7721 cells (Figures 5B, 5C). We found that EMT markers, including E-cadherin (CDH1), Vimentin (VIM), Snail, and $\mathrm{N}$-cadherin (CDH2), exhibited a substantial alteration in the differentially expressed genes. Considering that EMT is a metastatic cell event that increases cancer cell motility and malignant tumor progression [15], we performed RT-qPCR and western blotting to analyze the expression of EMT markers in both EMD-knocked down SMMC-7721 and HCC-LM3 cells. We demonstrated that silencing of EMD downregulated the E-cadherin (E-CAD), and upregulated Vimentin (VIM), Snail, and N-cadherin (N-CAD) in both SMMC-7721 and HCC-LM3 cells when compared with those in control cells (Figures 5D-5F), which was consistent with the data from RNA-seq. These findings suggest that EMD knockdown induces migration, invasion, and tumor metastasis by promoting EMT of HCC cells.

Interestingly, through analyzing the transcriptome data in RNA-seq, we noticed that p 21 mRNA (encoded by CDKN1A) was dramatically upregulated upon EMD knockdown in SMMC-7721 cells. Our RT-qPCR and western blotting further confirmed the upregulation of p21 at the mRNA and protein level in both EMD-knocked down SMMC-7721 and HCC-LM3 cells (Figures 5G-5I), which implies that upregulation of p21 may be involved in the regulation of cell motility, EMT, and tumor metastasis induced by EMD knockdown in HCC.

EMD knockdown induced EMT by upregulating the cytoplasmic p21 in HCC cells. Initially, p21 was considered as a tumor suppressor based on its ability to mediate cellcycle arrest, however, accumulating evidence indicates that cytoplasmic 21 protein might function as an oncogene and play a role in promoting cell motility and tumor progression [16-18]. Therefore, we questioned whether silencing of EMD increased migration, invasion, EMT, and tumor metastasis due to the cytoplasmic localization of p21 induced by EMD knockdown in HCC cells. As expected, EMD knockdown decreased the distribution of p21 in the nucleus and obviously increased the localization of p21 in the cytoplasm in both SMMC-7721 and HCC-LM3 cells (Figures 6A-6C). Moreover, we transfected SMMC-7721 and HCC-LM3 cells with specific siRNA against p21 or control (si-C), and the efficiency of p21 knockdown was confirmed in the si-p21 transfected cells (Figures 6D). Transwell analysis confirmed that silencing of p21 in both EMD-knocked down SMMC-7721 and HCC-LM3 cells significantly decreased the migratory ability and invasiveness of HCC cells when compared with those of control cells (Figures 6E, 6F). We further showed that silencing of p21 in EMD- knocked down SMMC-7721 and HCC-LM3 cells obviously increased the number of stress fibers and decreased the formation of lamellipodia (Figure 6G). In addition, the western blotting analysis further demonstrated that silencing of p21 partially reversed EMD knockdown-mediated induction of EMT in both SMMC-7721 and HCC-LM3 cells (Figure 6H). Collectively, these results suggest that upregulation of the cytoplasmic p21 induced by EMD knockdown is involved in the regulation of the migration, invasion, and EMT of HCC cells.

\section{Discussion}

Emerging evidence has demonstrated that EMD is involved in tumor cell motility and metastasis $[5,19]$, while the involvement of EMD in HCC metastasis has not been explored. In the present study, we demonstrated a significant role of EMD knockdown in regulating HCC cell migration and invasion and metastasis through upregulating the cytoplasmic p21, suggesting that EMD might represent a potential therapeutic target in HCC.

EMD has been shown to suppress cell motility and ERK pathway activation, and the loss of EMD results in nuclear instability and promotes cancer aggressiveness [6]. In the present study, we first examined the expression of EMD in HCC samples and found that EMD displays a trend of increased expression in HCC tissues. We also evaluated the expression of EMD in a panel of HCC cell lines with different spontaneous metastatic potential and demonstrated the relatively low expression of EMD in MHCC-97H, HCC-LM3, and SK-HEP1 cells with high metastatic potential and abundant expression of EMD in the HepG2, SMMC-7721, and Hep3B cells with low metastatic potential, suggesting that the EMD expression was negatively correlated with the metastatic potential. Meanwhile, we demonstrated that the loss of EMD in HCC cells facilitated cell migration and invasion in vitro and metastasis in vivo. It has been reported that EMD functions with its partners to polarize actin flow and cytoplasmic polarity [20]. Here, we further showed that EMD knockdown led to fewer stress fibers and more lamellipodia at the leading edge in HCC cells, and thereby contributed to the promotion of cell migration and invasion, indicating that EMD knockdown might be involved in modulating the actin dynamics and subsequently increase HCC cell motility.

To better understand the role of EMD knockdowninduced migration and invasion of HCC cells, RNA-seq analysis was performed to ascertain the altered expression of mRNA transcripts induced by EMD knockdown. A total of 1,132 differentially expressed genes were shown in EMD-knocked down SMMC-7721 cells, and GO and KEGG analysis revealed that the differentially expressed genes were enriched in the modulation of cell motility, actin cytoskeleton, and in various pathways. Among the differentially expressed genes, EMT markers exhibited remarkable changes. As is known, EMT is a metastatic event, which facilitates tumor cell migration, invasion, and metastasis. Then, we examine the effect of EMD knockdown on the expression of EMT markers in HCC cells. We revealed consistent significant downregulation of E-cadherin and upregulation of N-cadherin, Snail, and Vimentin in both EMD-knocked down SMMC-7721 and HCC-LM3 cells, indicating that 


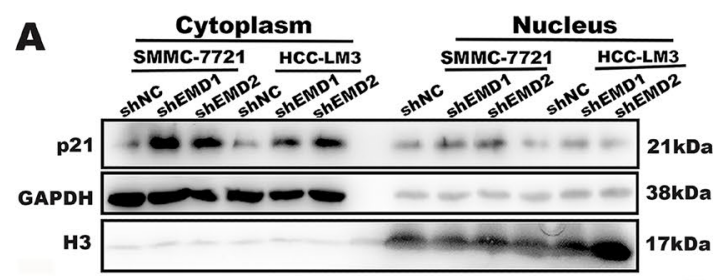

C

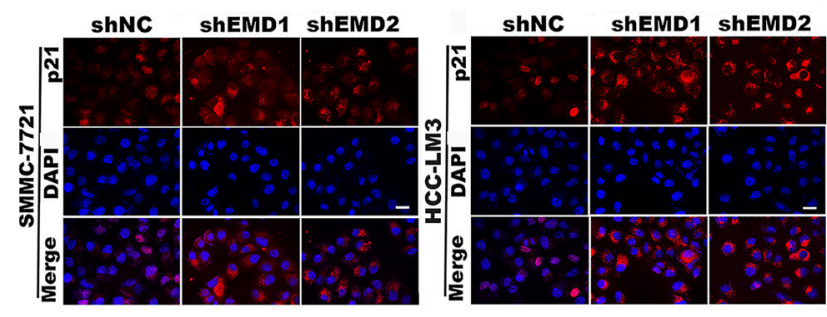

E

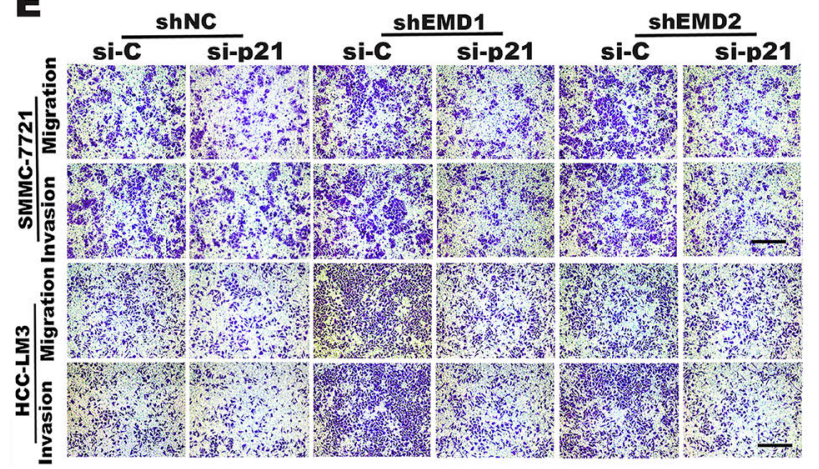

G

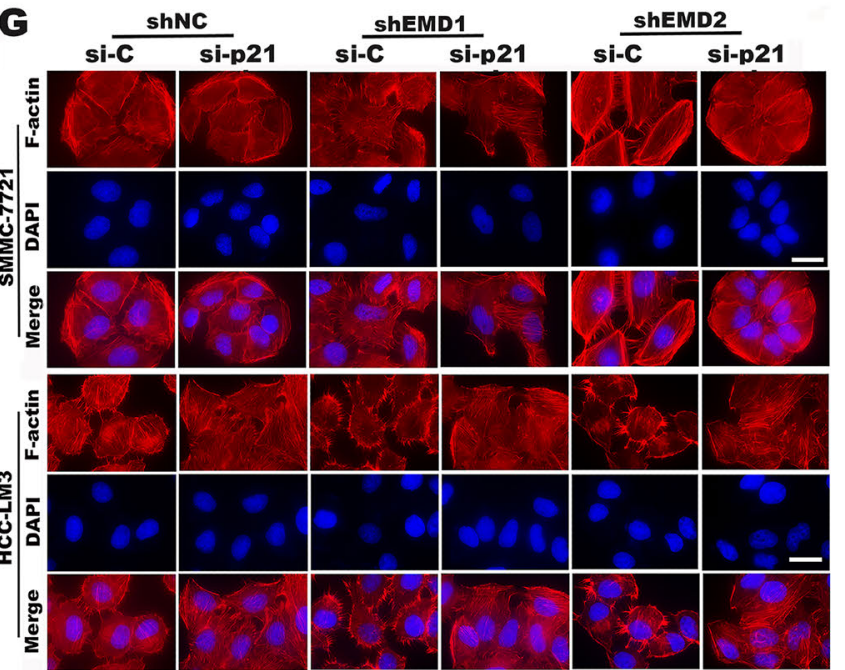

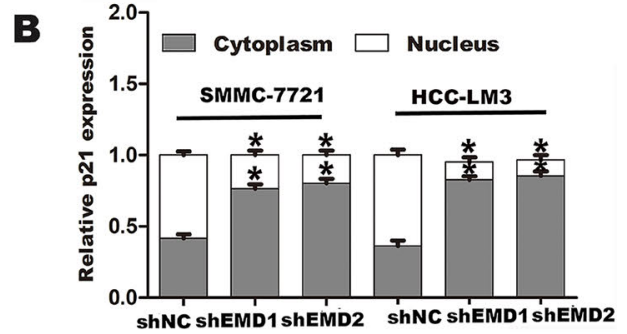

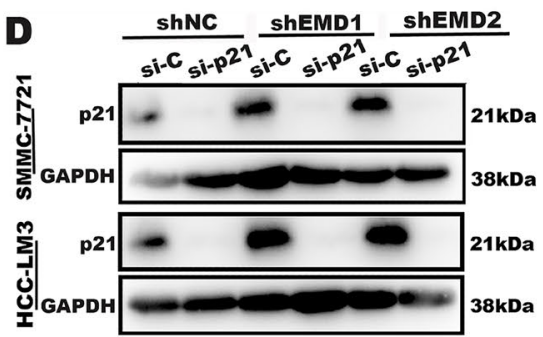

$F$ shNC+si-c $\square$ shNC+si-p21

$\square$ shEMD1+si-c shEMD1+si-p21

${ }^{2.0} \square$ shEMD2+si-c $\square$ shEMD2+si-p21

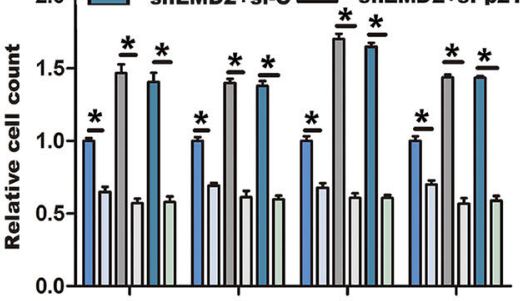

Migration Invasion Migration Invasion $\overline{\text { SMMC-7721 }}$

HCC-LM3

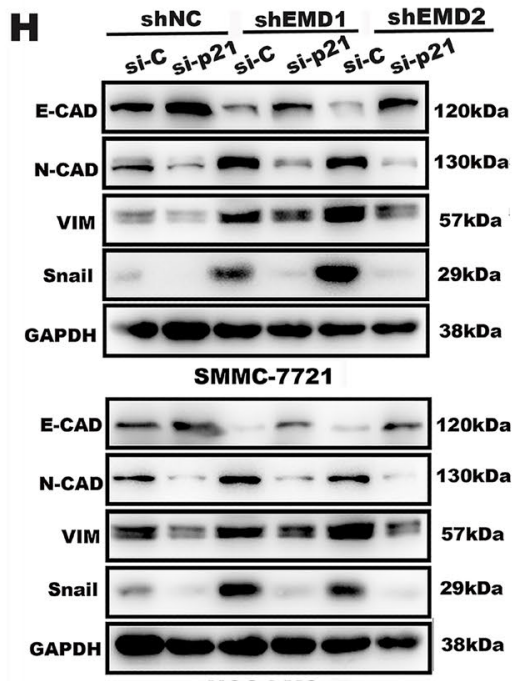

HCC-LM3

Figure 6. EMD knockdown induced EMT by upregulating the cytoplasmic p21 in HCC cells. A, B) Western blot analysis of p21 protein in the nucleus and cytoplasm of SMMC-7721 and HCC-LM3 cells upon EMD knockdown (A), and quantification of the relative protein levels of p21 in nuclear/cytoplasmic fraction is shown (B). GAPDH and H3 served as loading controls for cytosolic and nuclear fraction, respectively. Data presented as the mean \pm SD of three independent experiments. ${ }^{\star} \mathrm{p}<0.05$, vs. the indicated shNC. C) Representative images of immunofluorescence staining of p21 in EMDknocked down SMMC-7721 and HCC-LM3 cells are shown. Scale bar; $20 \mu \mathrm{m}$. D) The knockdown efficiency of p21 in EMD-knocked down SMMC-7721 and HCC-LM3 cells after transfection with specific siRNA against p21 (si-p21) and control (si-C) was tested by western blotting. GAPDH served as a loading control. E, F) Representative Transwell cell migration and invasion assays in EMD-knocked down SMMC-7721 and HCC-LM3 cells after silencing of p21 (E, scale bar; $100 \mu \mathrm{m})$, and quantification of relative numbers of migrated and invaded cells $(\mathrm{F})$ is shown. Data presented as the mean \pm SD of three independent experiments. ${ }^{*} \mathbf{p}<\mathbf{0 . 0 5}$, vs. the indicated control. G) Representative images of the actin cytoskeleton and DAPI staining in EMD-knocked down SMMC-7721 and HCC-LM3 cells after silencing of p21. Scale bar; $20 \mu \mathrm{m}$. H) Protein levels of EMT markers were determined by western blot in EMD-knocked down SMMC-7721 and HCC-LM3 cells upon silencing of p21. GAPDH served as a loading control. 
EMD knockdown induced HCC cell motility and metastasis by increasing the EMT process.

As the founding member of cyclin-dependent kinase inhibitors, p21 protein has primarily been described as a tumor suppressor based on its ability to block cancer cell proliferation. However, emerging evidence has demonstrated its dual role as a tumor suppressor or as an oncogene depending largely on its subcellular localization [21]. The p21 protein serving function as oncogenic potential often attributed to its cytoplasmic localization, thereby promoting cell motility $[21,22]$. In the current study, we found that, in agreement with the RNAseq findings, EMD knockdown dramatically upregulated the expression of $\mathrm{p} 21$ at both $\mathrm{mRNA}$ and protein levels in SMMC-7721 and HCC-LM3 cells. Notably, we further revealed that silencing of EMD obviously increased the localization of p21 in the cytoplasm in both SMMC-7721 and HCC-LM3 cells. To evaluate whether silencing of EMD induced migration, invasion, and EMT of HCC cells is contributed by upregulating p 21 in HCC cells, Transwell and immunofluorescence assays were then utilized to evaluate the cell migration and invasion upon p21 knockdown. The results showed that $\mathrm{p} 21$ knockdown reduced the migratory ability and invasiveness and regulated the actin cytoskeleton rearrangement in both EMD-knocked down SMMC-7721 and HCC-LM3 cells. Furthermore, western blot analysis revealed that knockdown of p21 partially reversed the expression of EMT markers induced by EMD knockdown in both SMMC-7721 and HCC-LM3 cells. Considering that EMD knockdown induced the phosphorylation of ERK and AKT, increased endogenous Notch signaling in cancer cells [6], the underlying mechanism as to how EMD knockdown modulates the expression of p21 needs to be further clarified. Taken together, these findings implied that silencing of EMD induced migration, invasion, and EMT could be contributed at least mediated by upregulating the cytoplasmic p 21 in HCC cells.

In summary, our results demonstrate that EMD knockdown increased migration, invasion, EMT, and metastasis of HCC cells by upregulating the cytoplasmic p21. Further study is required to address the underlying mechanisms as to whether cytoplasmic p21 participates in the modulation of EMT and metastasis in HCC cells directly or indirectly, and whether EMD knockdown induces p21 expression or regulates other pathways to promote HCC metastasis, however, our findings demonstrate that EMD may serve as not only a potential therapeutic target but also a novel potential biomarker for HCC patients.

Acknowledgments: This work was supported by the National Nature Science Foundation of China (no. 81672336), and the Training Program of Innovation and Entrepreneurship for College Students in Jiangsu Province (no. 202011117048Z and 202011117056Y). The Strengthening Health Care via Science and Education Project and Clinical Medical Innovation Platform Foundation of Yangzhou (2018 YXZX20184, Gastroenterology).

\section{References}

[1] TAGLIAMONTE M, MAURIELLO A, CAVALLUZZO B, RAGONE C, MANOLIO C et al. Tackling hepatocellular carcinoma with individual or combinatorial immunotherapy approaches. Cancer Lett 2020; 473: 25-32. https://doi. org/10.1016/j.canlet.2019.12.029

[2] PINATO DJ, GUERRA N, FESSAS P, MURPHY R, MINEO $\mathrm{T}$ et al. Immune-based therapies for hepatocellular carcinoma. Oncogene 2020; 39: 3620-3637. https://doi.org/10.1038/ s41388-020-1249-9

[3] HUANG Z, ZHOU JK, WANG K, CHEN H, QIN S, et al. PDLIM1 Inhibits Tumor Metastasis Through Activating Hippo Signaling in Hepatocellular Carcinoma. Hepatology 2020; 71: 1643-1659. https://doi.org/10.1002/hep.30930

[4] HYUN MH, LEE YS, KIM JH, LEE CU, JUNG YK et al. Hepatic resection compared to chemoembolization in intermediate- to advanced-stage hepatocellular carcinoma: A meta-analysis of high-quality studies. Hepatology 2018; 68: 977-993. https://doi.org/10.1002/hep.29883

[5] REIS-SOBREIRO M, CHEN JF, NOVITSKAYA T, YOU S, MORLEY S et al. Emerin Deregulation Links Nuclear Shape Instability to Metastatic Potential. Cancer Res 2018; 78: 60866097. https://doi.org/10.1158/0008-5472.CAN-18-0608

[6] LEE B, LEE TH, SHIM J. Emerin suppresses Notch signaling by restricting the Notch intracellular domain to the nuclear membrane. Biochim Biophys Acta Mol Cell Res 2017; 1864: 303-313. https://doi.org/10.1016/j.bbamcr.2016.11.013

[7] HO CY, JAALOUK DE, VARTIAINEN MK, LAMMERDING J. Lamin A/C and emerin regulate MKL1-SRF activity by modulating actin dynamics. Nature 2013; 497: 507-511. https://doi.org/10.1038/nature12105

[8] NASTAŁY P, PURUSHOTHAMAN D, MARCHESI S, POLI A, LENDENMANN $\mathrm{T}$ et al. Role of the nuclear membrane protein Emerin in front-rear polarity of the nucleus. Nat Commun 2020; 11: 2122. https://doi.org/10.1038/s41467020-15910-9

[9] DILSAVER MR, CHEN P, THOMPSON TA, REUSSER T, MUKHERJEE RN et al. Emerin induces nuclear breakage in Xenopus extract and early embryos. Mol Biol Cell 2018; 29: 3155-3167. https://doi.org/10.1091/mbc.E18-05-0277

[10] HOLASKA JM, KOWALSKI AK, WILSON KL. Emerin caps the pointed end of actin filaments: evidence for an actin cortical network at the nuclear inner membrane. PLoS Biol 2004; 2: E231. https://doi.org/10.1371/journal.pbio.0020231

[11] ROWAT AC, LAMMERDING J, IPSEN JH. Mechanical properties of the cell nucleus and the effect of emerin deficiency. Biophys J 2006; 91: 4649-4664. https://doi. org/10.1529/biophysj.106.086454

[12] ABDOU AG, ABDELWAHED M, SAID A, TAIE DM, FAHMY S. Evaluation of the diagnostic value of emerin and CD56 in papillary thyroid carcinoma - an immunohistochemical study. J Immunoassay Immunochem 2018; 39: 521-537. https://doi.org/10.1080/15321819.2018.1514508

[13] XU X, ZHOU W, CHEN Y, WU K, WANG H et al. Immediate early response protein 2 promotes the migration and invasion of hepatocellular carcinoma cells via regulating the activity of Rho GTPases. Neoplasma 2020; 67: 614-622. https://doi.org/10.4149/neo_2020_190818N781 
[14] SHEN WG, XUE QY, ZHU J, HU BS, ZHANG Y, et al. Inhibition of adenovirus-mediated human MAGE-D1 on angiogenesis in vitro and in vivo. Mol Cell Biochem 2007; 300: 89-99. https://doi.org/10.1007/s11010-006-9373-6

[15] YANG J, WEINBERG RA. Epithelial-mesenchymal transition: at the crossroads of development and tumor metastasis. Dev Cell 2008; 14: 818-829. https://doi.org/10.1016/j.devcel.2008.05.009

[16] ABBAS T, DUTTA A. p21 in cancer: intricate networks and multiple activities. Nat Rev Cancer 2009; 9: 400-414. https:// doi.org/10.1038/nrc2657

[17] COQUERET O. New roles for p21 and p27 cell-cycle inhibitors: a function for each cell compartment? Trends Cell Biol 2003; 13: 65-70. https://doi.org/10.1016/s09628924(02)00043-0

[18] ROMANOV VS, POSPELOV VA, POSPELOVA TV. Cyclin-dependent kinase inhibitor p21(Waf1): contemporary view on its role in senescence and oncogenesis. Biochemistry (Mosc) 2012; 77: 575-584. https://doi.org/10.1134/ S000629791206003X
[19] RANADE D, PRADHAN R, JAYAKRISHNAN M, HEGDE S, SENGUPTA K. Lamin A/C and Emerin depletion impacts chromatin organization and dynamics in the interphase nucleus. BMC Mol Cell Biol 2019; 20: 11. https://doi. org/10.1186/s12860-019-0192-5

[20] CHANG W, FOLKER ES, WORMAN HJ, GUNDERSEN GG. Emerin organizes actin flow for nuclear movement and centrosome orientation in migrating fibroblasts. Mol Biol Cell 2013; 24: 3869-3880. https://doi.org/10.1091/mbc.E1306-0307

[21] KREIS NN, LOUWEN F, YUAN J. The Multifaceted p21 (Cip1/Waf1/CDKN1A) in Cell Differentiation, Migration and Cancer Therapy. Cancers (Basel) 2019; 11: 1220. https:// doi.org/10.3390/cancers11091220

[22] LEE S, HELFMAN DM. Cytoplasmic p21Cip1 is involved in Ras-induced inhibition of the ROCK/LIMK/cofilin pathway. J Biol Chem 2004; 279: 1885-1891. https://doi.org/10.1074/ jbc.M306968200 